\title{
Uso de plantas nativas para la rehabilitación de canteras en Sonora
}

\author{
Use of Native Plants for the Rehabilitation of Quarries in Sonora
}

\author{
Diana Miriam Mc Caughey-Espinoza ${ }^{1 *}$, Gloria Irma Ayala-Astorga1, M. Guadalupe Burboa-Zazueta1, \\ Rafael Retes-López², Andrés Ochoa-Meza²
}

\begin{abstract}
RESUMEN
El estado de Sonora no es ajeno a los problemas ocasionados por el calentamiento global, tales como la disminución de poblaciones de especies nativas debido a actividades como el cambio de uso de suelo para la explotación de áridos (rocas), y las escasas o nulas precipitaciones que se presentan. En este trabajo se utilizaron 4 especies de árboles nativos (mezquite, Prosopis velutina; palo verde chino, Parkinsonia microphylla; palo verde azul, Parkinsonia florida y palo fierro, Olneya tesota), para la rehabilitación de canteras. El porcentaje de germinación de las semillas fue de 95 a 99\% en general. Se trasplantaron diez ejemplares de cada especie. La tasa de sobrevivencia al trasplante a los 15 días fue de $100 \%$ y al mes de 80 a 90\%. Los datos corresponden a la evaluación 8 años después del trasplante, donde la altura alcanzada por el mezquite fue 2,91 metros, palo verde chino 2,67 metros, palo verde azul 2,56 metros y palo fierro 1,13 metros. La cobertura de copa en mezquite fue $12,315 \mathrm{~cm}^{2}$, palo verde chino $9,913 \mathrm{~cm}^{2}$, palo verde azul $9.423 \mathrm{~cm}^{2}$ y palo fierro $3.175 \mathrm{~cm}^{2}$. La cobertura de tallo en mezquite fue $98,2 \mathrm{~cm}^{2}$, palo verde chino $97,4 \mathrm{~cm}^{2}$, palo verde azul $96.4 \mathrm{~cm}^{2}$ y palo fierro $43,4 \mathrm{~cm}^{2}$, mientras que el índice de crecimiento en altura fue de 56.00 a $82,76 \%$ y el diámetro de copa de 99,60 a 99,86\%. Por último, el diámetro de tallo fue de 97,74 a 99,07\%.
\end{abstract}

Palabras clave: plantas nativas, trasplante, sobrevivencia, germinación

\begin{abstract}
The Sonora state is not exempted of global warming consequences, such as the reduction of native species populations by activities like as land use change for the exploitation of aggregates (rocks) and the scarce or no rainfalls events occurred. In this work 4 species of native trees were used (mesquite, Prosopis velutina, chinese green stick, Parkinsonia microphylla, blue green stick, Parkinsonia florida and ironwood, Olneya tesota), for the rehabilitation of quarries. The germination percentage of the seeds was 95 to $99 \%$ in general. Ten specimens of each species were transplanted, the transplantation survival rate at 15 days was $100 \%$ and at $80-90 \%$ at one month. The data corresponds to the evaluation at 8 years after the transplant, where the height reached by the mesquite was 2.91 meters, chinese green stick 2.67 meters, blue green stick 2.56 meters and ironwood 1.13 meters. Mesquite cup cover was $12,315 \mathrm{~cm}^{2}$, chinese green stick $9,913 \mathrm{~cm}^{2}$, blue green stick $9,423 \mathrm{~cm}^{2}$ and ironwood with $3,175 \mathrm{~cm}^{2}$, mesquite stem coverage was $98.2 \mathrm{~cm}^{2}$, chinese green stick $97.4 \mathrm{~cm}^{2}$, blue green stick $96.4 \mathrm{~cm}^{2}$ and Ironwood $43.4 \mathrm{~cm}^{2}$, while the height growth index was 56.00 to $82.76 \%$ and the crown diameter was 99.60 to $99.86 \%$, finally, stem diameter was 97.74 to $99.07 \%$.
\end{abstract}

Key words: native plants, transplant, survival, germination

\section{Introducción}

Sonora es un estado semiárido, en el cual existen diversas actividades muy importantes, como la agricultura, ganadería, pesca y minería. En este último sector se puede señalar a las empresas dedicadas a la producción de cemento, las cuales trabajan a cielo abierto y la primera etapa es la explotación de rocas, lo

1 Departamento de Investigaciones Científicas y Tecnológicas de la Universidad de Sonora. Hermosillo Sonora, México.

2 Departamento de Agricultura y Ganadería. Universidad de Sonora. Hermosillo Sonora, México.

* Autor de correspondencia: diana.mccaughey@unison.mx 
que conlleva la extracción minera de materia prima de la corteza terrestre. Dicho proceso implica impactos inevitables sobre las áreas naturales existentes. En particular, la remoción de tierra y los cambios en la topografía tienden a afectar ecosistemas locales, en los cuales habitan animales silvestres y existen fuentes de agua (Bos y Mastoris, 2011).

De dichos sitios también se extraen rocas industriales y ornamentales. Al ir explotando estas áreas, se van formando las canteras, en las cuales existe baja o nula presencia de suelo y materia orgánica, por lo tanto, no se da la oportunidad de regenerar algún tipo de vegetación de modo natural. Esto ha contribuido de una u otra manera al calentamiento global, al formarse islas de calor en lugares donde anteriormente existía vegetación nativa con gran diversidad, constituida principalmente por plantas maderables y no maderables, como mezquite (Prosopis velutina Wooton), palo verde chino (Parkinsonia microphyllum Torr), sahuaro (Carnegiea gigantea Britton \& Rose), palo verde azul (Parkinsonia florida Benth), agave bacanora (Agave angustifolia Haw), lechuguilla (Agave lechuguilla Torr) y palo fierro (Olneya tesota A. Gray).

El palo fierro se encuentra sujeto a protección especial y el sahuaro está en la categoría de las especies amenazadas de acuerdo a la Norma Oficial Mexicana NOM-059-SEMARNAT-2010 (México, 2010). Para minimizar el impacto que se ocasiona al ecosistema, se planteó establecer especies nativas que presentaran mínimos problemas para su propagación, manejo, trasplante y adaptación a través de acciones ecológicas que permitan cambiar el contorno de un área perturbada a otra con posibilidades de volver a generar vida de flora y fauna silvestre, así como atenuar el efecto invernadero que va avanzando con las actividades y origina lo que actualmente se conoce como la huella ecológica personal.

\section{Materiales y métodos}

\section{Ubicación del área de estudio}

El presente trabajo de investigación se llevó a cabo en el mes de julio de 2010, en la cantera de una planta cementera localizada en el Km 23 de la carretera Hermosillo a Sahuaripa, Sonora, México, la cual se encuentra en $29^{\circ} 05^{\prime} 42.79^{\prime \prime}$ latitud norte y $110^{\circ} 71^{\prime} 94.03$ " longitud oeste, con una altitud media de $290 \mathrm{msnm}$, una precipitación media anual de 330 mm y temperatura promedio de $24{ }^{\circ} \mathrm{C}$, con un tipo de vegetación denominado matorral arbosufrutescente. Ocasionalmente se tienen heladas y granizadas en el invierno.

Área seleccionada: para la realización de este trabajo se seleccionó un área de 240 metros cuadrados aproximadamente de cantera, que se localiza en la parte Noreste de la planta. El suelo es rocoso litosol, escaso de materia orgánica (Figura $1)$.

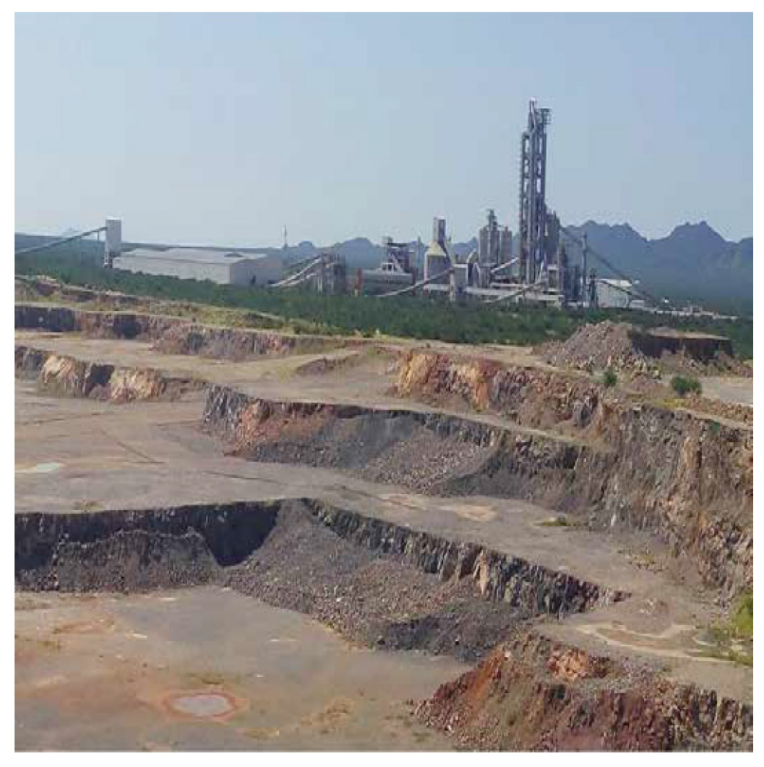

Figura 1.- Área de estudio: Cantera 
Especies en estudio: para la realización de este estudio se seleccionaron especies endémicas del estado de Sonora: palo fierro (Olneya tesota A. Gray), mezquite (Prosopis velutina Wooton), palo verde chino (Parkinsonia microphyllum (Torr) Rose y I. M. Johnst) y palo verde azul (Parkinsonia florida (Benth. ex A.Gray) S. Wats). Estas plantas fueron producidas a partir de semillas en condiciones de vivero cercano al área de estudio.

Colecta de semillas: el material vegetativo que se utilizó para la propagación sexual de las especies se recolectó de las plantas arbóreas más cercanas al sitio de estudio en el año 2009. Las semillas de mezquite y ambos palos verdes se extrajeron de la péchita (vaina), mientras que las semillas de palo fierro se recolectaron del dosel de los árboles. Posteriormente las semillas de las cuatro especies en estudio se colocaron en una temperatura de $4{ }^{\circ} \mathrm{C}$, para evitar el daño por insectos (Figura 2).

Tratamiento pregerminativo: las semillas de palo fierro se remojaron por 24 horas en agua a temperatura ambiente, mientras que a las semillas de mezquite y ambos palos verdes se les realizó una escarificación fisicoquímica utilizando agua a una temperatura de $80{ }^{\circ} \mathrm{C}$ durante tres minutos y posteriormente se dejaron remojando por 24 horas.
Siembra: una vez preparadas las semillas para efectuar la siembra, se sembraron en bolsas negras de polietileno de una capacidad de un kg de tierra aproximadamente. El sustrato que se utilizó fue una mezcla de 50\% arcilla y 50\% arena, para asegurar un buen drenaje y evitar así la presencia de hongos en el suelo y por ende pudrición de semillas. Para ello se utilizaron 100 semillas de cada especie y se colocó una semilla por bolsa (maceta).

Preparación de terreno: se realizaron perforaciones en el suelo de la cantera con una profundidad promedio de $40 \mathrm{~cm}$ y $50 \mathrm{~cm}$ de ancho, colocándoles materia orgánica (hojarasca $\mathrm{y}$ arcilla), con la finalidad de que las plantas siguieran produciendo más raíces y estas a su vez aseguraran su establecimiento en la cantera.

Trasplante y distribución: las plantas tenían una edad de cuatro meses y una altura promedio de $48,5 \mathrm{~cm}$. Se utilizaron 10 plantas por especie y se trasplantaron en la cantera en el mes de agosto. La distancia que se manejó entre plantas fue de seis metros. Las plantas se sacaron de la bolsa negra de polietileno y se colocaron sobre la mezcla de arcilla y hojarasca. Posteriormente se les colocó más arcilla, se presionó la tierra con la finalidad de sacar todo el aire presente y evitar que las raíces se
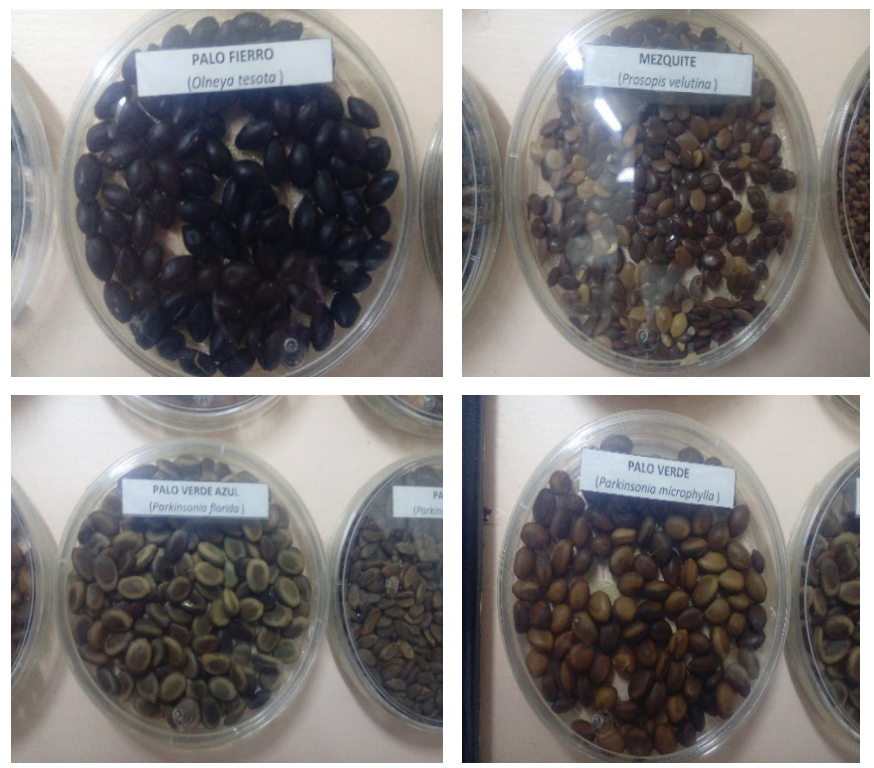

Figura 2.-Semillas de las especies utilizadas. 
secaran y por último, se les añadió agua (Figura 3). La distribución de las especies en el sitio de estudio fue al azar.

Riegos: se aplicaron tres riegos pesados durante los primeros tres días, con aproximadamente 10 litros por planta y posteriormente una vez por semana con el mismo volumen durante 6 meses. Así se obliga a las plantas para que se adapten en el área y a que generen más raíces.

Variables evaluadas: para determinar el porcentaje de germinación de las semillas se siguió el método de la Asociación Internacional de Ensayos de Semillas (ISTA, 1996), utilizando 100 semillas de cada especie. Se registró el porcentaje de germinación al término del ensayo.

Para conocer el comportamiento de las plantas en este tipo de áreas perturbadas, donde está presente una fuente de calor constante durante todo el día, lo que implica una situación de continuo estrés, se tomó la decisión de evaluar la sobrevivencia a los 15 y 30 días después de su trasplante, mientras que la altura, diámetro de copa y diámetro de tallo, al inicio y a los 8 años después de su trasplante. Con ello se calculó el porcentaje de crecimiento en este lapso de las especies existentes en el estudio.

Las mediciones de altura, diámetro de copa y tallo se llevaron a cabo en cada individuo para obtener la medición dasométrica (Meza y Osuna, 2003). Para ello se utilizó una cinta métrica expresada en centímetros o metros, un vernier digital modelo CD6" CSX (Mitutoyo Corp, Japan).
El porcentaje de crecimiento o índice de crecimiento se determinó de acuerdo a la metodología de Carrillo et al. (2007), usando la siguiente ecuación:

Donde:

$$
\mathrm{IC}=\frac{\mathrm{Xf}-\mathrm{Xi} * 100}{\mathrm{Xf}}
$$

$\mathrm{IC}=$ Índice de crecimiento en $\%$

$\mathrm{Xf}=$ Valor obtenido al final del período para altura, diámetro de copa o área basal.

$\mathrm{Xi}=$ Valor obtenido al inicio del período para altura, diámetro de copa o área basal.

Para las mediciones dasométricas y el índice de crecimiento se consideró solo a los individuos que sobrevivieron a los 8 años después de su trasplante.

Por último, se consideró monitorear el comportamiento climatológico de algunos parámetros en promedio anual, como fueron las temperaturas máximas y mínimas, al igual que la presentación y el porciento de humedad relativa durante el periodo de 2010 a 2018, utilizando un medidor y registrador Data logger Modelo WK057.

\section{Resultados y discusión}

Porcentaje de germinación: al evaluar el porcentaje de germinación de cada una de las especies, se obtuvo un $99 \%$ para palo fierro y un $95 \%$ para el resto de las especies. La germinación se inició al tercer día de la siembra, lo cual garantiza
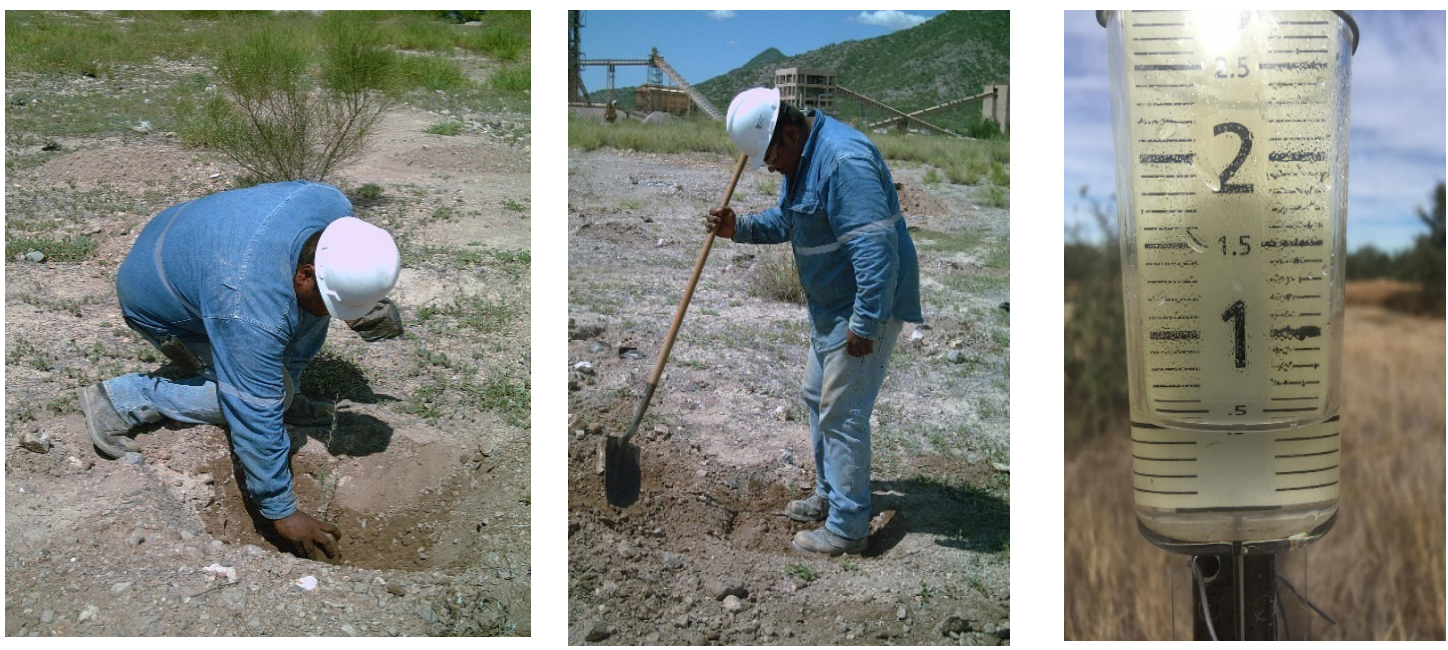

Figura 3.-Trasplante de las especies y medición de la precipitación pluvial. 
que dichas especies de plantas presentan un excelente vigor germinativo, y esto permite contar con nuevos individuos para la conservación de la flora de esta región. Sobrevilla et al. (2013) evaluaron diferentes tratamientos de escarificación en mezquite (Prosopis laevigata), utilizando una lija manual, y obtuvieron 25\% de germinación. Quiñónez et al. (2013) evaluaron diferentes tratamientos en semillas de mezquite $P$. laevigata y obtuvieron $70 \%$ de germinación cuando se sumergieron las semillas en agua a una temperatura de $70{ }^{\circ} \mathrm{C}$ durante 8 minutos. Por su parte Villarreal et al. (2013) evaluaron la germinación in vitro de semillas de mezquite Prosopis glandulosa Torr. utilizando ácido sulfúrico, y las sumergieron durante 20 minutos para obtener $96 \%$ de germinación. RuedaPuente et al. (2017) obtuvieron del 96 al 100\% de germinación al utilizar un inoculante bacterial de Rhodococcus fascians y Azospirillum halopraeferens en condiciones de invernadero. Sañudo-Torres et al. (2009) lograron 70,3\% de germinación y utilizaron la escarificación con lija en semillas de palo fierro (Olneya tesota).

Sobrevivencia al trasplante: no se realizaron labores agronómicas como el control de malezas, debido a que mitigan la erosión del suelo, sobre todo de estas áreas. Las plantas son individuos capaces de adaptarse, siempre y cuando tengan las condiciones básicas, como son agua y nutrientes (Baur, 2004). El comportamiento de adaptación y sobrevivencia al trasplante de las plantas arbóreas fue excelente, al mostrar un $100 \%$ de sobrevivencia a los 15 días del trasplante de las cuatro especies en estudio y $80 \%$ de sobrevivencia al mes en plantas de palo fierro, mientras que el mezquite y ambos palos verdes presentaron $90 \%$ de sobrevivencia. La sobrevivencia que se registró garantiza a mediano o largo plazo recuperar parte de la vegetación anteriormente existente en sitios como las canteras, ya que la vegetación que pudiera presentarse de manera espontánea es limitada por la acumulación de limo acarreado por el aire o lluvias y por la deposición de semillas de especies efímeras, las cuales forman un mantillo que cubre estas áreas año tras año pero solo por un corto tiempo, para después volver el suelo a quedar desnudo.

El porcentaje de sobrevivencia al trasplante es similar al obtenido por Mc Caughey-Espinoza et al. (2017), donde para las mismas especies se logró 90\% de sobrevivencia, en condiciones menos adversas que aquellas a las que fueron sometidas en el presente estudio. Quiñónez et al. (2013) obtuvieron 90\% de sobrevivencia al aplicar 10 litros de agua cada mes en las plantas de Prosopis laevigata, lo que sugiere que las especies utilizadas en este estudio tienen demandas básicas de humedad para poder sobrevivir, es decir, no requieren de mucha humedad siempre y cuando su trasplante sea antes de las lluvias o en la época de lluvias. Por otra parte, se reconoce que el trasplante en la época de lluvias hace que dichas plantas sean menos propensas a ser consumidas por la fauna silvestre.

Comportamiento de las especies: para este apartado se registró la altura, diámetro basal, diámetro de copa y el porcentaje de crecimiento de cada uno de estos parámetros.

Con respecto a la altura promedio de las especies en estudio, después de los 8 años de su trasplante, el mezquite presentó la mayor altura con 2,91 metros, seguido del palo verde chino con 2,67 metros, palo verde azul 2,56 metros y palo fierro 1,13 metros (Tabla 1). Ríos et al. (2011) reportan alturas que varían desde 7 hasta $27 \mathrm{~cm}$ al trasplante, lo que contrasta con el punto inicial de $48,5 \mathrm{~cm}$ y que atiende a las recomendaciones de los mismos autores para incrementar la sobrevivencia. De acuerdo a los resultados obtenidos por García et al. (2014), el mezquite (Prosopis glandulosa Torr.) de una edad de 11 años presentó una altura promedio de 3,7 metros. Mc Caughey-Espinoza et al. (2017) evaluaron el palo verde azul (Parkinsonia florida), que alcanzó un promedio de 1,97 metros; mezquite (Prosopis velutina) 1,93, palo verde chino (Parkinsonia microphyllum) 1,92 metros y palo fierro (Olneya tesota) 0.90 metros. Turner et al. (2005) evaluaron la altura en palo fierro (Olneya tesota A. Gray), el cual alcanzó los 5-10 metros, palo verde chino

Tabla 1. Promedio del comportamiento de las especies de árboles.

\begin{tabular}{|c|c|c|c|c|c|c|}
\hline \multirow{2}{*}{ Especies } & \multicolumn{3}{|c|}{ Trasplante } & \multicolumn{3}{|c|}{ Después del trasplante } \\
\hline & Altura $\mathrm{cm}$ & Cobertura de copa $\mathrm{cm}^{2}$ & Cobertura de tallo $\mathrm{cm}^{2}$ & Altura m & Cobertura de copa $\mathrm{cm}^{2}$ & Cobertura de tallo $\mathrm{cm}^{2}$ \\
\hline Mezquite & 50,16 & 16,29 & 0,91 & 2,91 & 12,315 & 98,2 \\
\hline Palo fierro & 49,72 & 12,68 & 0,98 & 1,13 & 3,175 & 43,4 \\
\hline Palo verde. a. & 48,87 & 15,19 & 0,91 & 2,56 & 9,423 & 96,7 \\
\hline Palo verde. c. & 49,19 & 15,81 & 0,97 & 2,67 & 9,913 & 97,4 \\
\hline
\end{tabular}


(Parkinsonia microphyllum) 6 metros y palo verde azul (Parkinsonia florida) más de 12 metros. Dichos resultados difieren de los obtenidos en este estudio, ya que las plantas de mezquite (Prosopis velutina), palo verde chino (Parkinsonia microphyllum), palo verde azul (Parkinsonia florida) y palo fierro (Olneya tesota) que se utilizaron tenían una edad de cuatro meses aproximadamente (en vivero) y 8 años en campo (trasplantadas). Mientras que Ríos et al. (2011) en mezquite (Prosopis laevigata (H. \& B.) Jonhst) mostraron alturas de 52,3-87,8 cm, lo cual evidencia que son plantas jóvenes y la medición fue a los 3 meses después del trasplante. Estos resultados son importantes, comparados con las alturas que Ríos et al. (2011) obtuvieron en mezquite. Las especies estudiadas, de acuerdo a sus características fisiológicas, presentaron un buen crecimiento, sobre todo la de palo fierro, ya que se considera una especie de lento crecimiento. Ello pudiera atribuirse a que en vivero esta planta puede desarrollarse más rápido que a nivel de campo hasta llegar a una edad en la cual requiera más espacio para su sistema radicular.

Diámetro de copa: el desarrollo foliar de estas especies de árboles se puede observar en la Tabla 1, donde el mezquite presentó mayor área de copa con $12,315 \mathrm{~cm}^{2}$, seguido del palo verde chino con 9.913 $\mathrm{cm}^{2}$ y palo verde azul con $9.423 \mathrm{~cm}^{2}$, mientras que palo fierro alcanzó $3.175 \mathrm{~cm}^{2}$. A pesar de ser plantas jóvenes y expuestas a condiciones adversas, mostraron un excelente diámetro de copa (cubierta vegetal). Dichos resultados son atribuibles a la disponibilidad de agua, lo que demuestra que la aplicación de riegos facilita su desarrollo. García et al. (2014), al medir el diámetro de copa en mezquites (Prosopis grandulosa Torr.) de 11 años de edad, obtuvieron un promedio de $17,88 \mathrm{~m}^{2}$, y al compararlos con los alcanzados en este estudio, el mezquite (Prosopis velutina Wooton) mostró un diámetro de copa de $22,3 \mathrm{~cm}^{2}$. Mc CaugheyEspinoza et al. (2017) evaluaron plantas con una edad de dos años y el mezquite (Prosopis velutina) presentó un diámetro de copa de $2,82 \mathrm{~m}^{2}$, palo verde chino (Parkinsonia microphyllum) 2,65 $\mathrm{m}^{2}$, palo verde azul (Parkinsonia florida) 2,58 $\mathrm{m}^{2}$ y palo fierro (Olneya tesota) $0,98 \mathrm{~m}^{2}$. Los resultados muestran una gran diferencia entre las especies de mezquites como consecuencia de la edad de las plantas.

Las plantas utilizadas en este estudio son consideradas como plantas del desierto sonorense y se ha determinado que requieren poca agua para satisfacer sus necesidades. Pero aun así, es de suma importancia la disponibilidad de agua para el establecimiento y crecimiento de las especies, ya que estas en su estado silvestre son de lento crecimiento, aunado al pastoreo de animales silvestres o domésticos, baja precipitación, prácticas de podas inmoderadas e incendios forestales. Esto genera un estrés en las plantas, pudiendo llegar en un momento dado a que se dañen y mueran (Mc Caughey-Espinoza et al., 2017), aunado a la explotación de ecosistemas para el cambio de uso de suelo, la presencia de plagas y enfermedades que día a día se están presentando y no se tenían contempladas para estas especies nativas en esos sitios semidesérticos. Es necesario considerar la importancia de los problemas que se presentan y que no se ven a simple vista, y se pueden tomar como problemas ecológicos silenciosos, "los cuales es necesario atender con oportunidad estudiando las alternativas que permitan proveer soluciones viables en el corto y mediano plazo".

Diámetro de tallo: para contar con árboles fuertes es necesario que presenten un buen sistema radicular, lo que contribuirá a tener árboles de buena talla, con un diámetro de copa apropiado y sobre todo un tallo firme, ya que es el pie de la planta y por lo tanto deberá soportar su peso. De las especies en estudio, el mezquite presentó mayor diámetro de tallo con un promedio de $98,2 \mathrm{~cm}^{2}$, seguido del palo verde chino con $97,4 \mathrm{~cm}^{2}$ y palo verde azul con $96,7 \mathrm{~cm}^{2}$, y por último, el palo fierro con $43,4 \mathrm{~cm}^{2}$, a pesar de ser plantas jóvenes y expuestas a condiciones adversas (Tabla 1).

Al comparar los resultados con los de Ríos et al. (2011) en mezquite Prosopis laevigata (H. \& B.) Jonhst, obtuvieron un promedio en la cobertura aérea de $8,63 \mathrm{~cm}^{2}$ a los 63 días después del trasplante utilizando $20 \mathrm{G}$ de hidrogel por planta. García et al., (2014) reportaron un diámetro promedio de 93.415 $\mathrm{cm}^{2}$ en mezquite (Prosopis glandulosa Torr.). Son árboles adultos y el área de plantación fue en una zona agrícola donde existen nutrientes, materia orgánica y la posibilidad de tener humedad cerca, por lo cual, los datos difieren de los obtenidos en este estudio. Mc Caughey-Espinoza et al. (2017) evaluaron plantas de dos años de edad, donde el mezquite (Prosopis velutina) presentó un diámetro de tallo de $30,68 \mathrm{~cm}^{2}$, palo verde chino (Parkinsonia microphyllum) 29,28 $\mathrm{cm}^{2}$, palo verde azul (Parkinsonia florida) $28,34 \mathrm{~cm}^{2}$ y palo fierro (Olneya Tesota) $7,45 \mathrm{~cm}^{2}$. Se consideran seis condiciones importantes para tener resultados deseables en el trasplante de las especies: que sean plantas de la región, agua disponible, tipo de suelo, nutrientes, época de trasplante y protección de la 
fauna silvestre o doméstica. Si se tienen en cuenta al menos las primeras tres, es posible esperar que el resultado del trasplante sea exitoso. A pesar de que en este trabajo solo se consideraron dos de estas, se obtuvieron resultados aceptables.

Índice de crecimiento: en este trabajo se observó un importante índice de crecimiento (Tabla 2) con respecto al diámetro de tallo. Mezquite presentó $99,07 \%$, palo verde azul 99,05\%, seguido del palo verde chino con $99,00 \%$ y por último palo fierro con $97,74 \%$. Esto podría considerarse como un porcentaje de crecimiento normal para cada una de las especies incluyendo a palo fierro. Con respecto al índice de crecimiento en el diámetro de copa, mostró un mayor porcentaje el mezquite con $99,86 \%$, seguido del palo verde chino con $99,84 \%$, palo verde azul $99,83 \%$ y palo fierro con $97,74 \%$. En los datos de altura, el mezquite presentó mejor porcentaje de crecimiento con $82,76 \%$, luego están el palo verde chino y azul con $81,57 \%$ y $80,91 \%$ y por último el palo fierro con 56,00\%. Mc Caughey-Espinoza et al. (2017) evaluaron el índice de crecimiento de algunas especies, de las cuales el palo verde azul (Parkinsonia florida) presentó el triple del crecimiento en la altura con un $82,5 \%$, y los que mostraron crecimiento similar fueron mezquite (Prosopis velutina) con $32,1 \%$, palo verde chino (Parkinsonia microphyllum) con 26,6\% y palo fierro (Olneya Tesota) con 23,3\%. Mientras que, en el diámetro de copa, el palo verde chino presentó el mejor índice de crecimiento con $48,7 \%$, palo verde azul $34,9 \%$, mezquite $33,0 \%$ y palo fierro $17,3 \%$. Por último, en el índice de crecimiento del tallo el mezquite mostró $23,7 \%$ más en el diámetro de tallo, seguido del palo verde azul con $21,8 \%$, palo verde chino con $21,7 \%$ y palo fierro con $17,9 \%$. Los resultados expuestos son favorables para este estudio, ya que proporcionan información relevante respecto al desarrollo de esas especies en estas áreas. Es importante señalar que este tipo de plantaciones contribuyen a mitigar el impacto del calentamiento global por el cambio de uso de suelo, lo que ha generado hornos latentes (canteras), y permite reducir la incidencia atenuando el calor de la radiación solar. Aunado a lo anterior, dichas especies mostraron capacidad para un amplio manejo y adaptación para áreas de este tipo en las cuales hay mucho movimiento de personal y maquinaria pesada, explosiones y las plantas se encuentran expuestas a ser consumidas por fauna silvestre, lo que implica una situación de estrés continuo. La vegetación que aún está presente puede desaparecer y con ella un sinfín de especies de animales y hasta tribus que existen en algunos lugares. La recopilación de información que contribuya a la discusión de los resultados, en algunos casos fue imposible, ya que son escasos los datos sobre este tipo de especies.

Tabla 2. Índice de crecimiento de las especies de árboles (\%).

\begin{tabular}{cccc}
\hline \multirow{2}{*}{ Especies } & \multicolumn{3}{c}{ Índice de crecimiento (\%) } \\
\cline { 2 - 4 } & Altura cm & $\begin{array}{c}\text { Cobertura de } \\
\text { copa cm }\end{array}$ & $\begin{array}{c}\text { Cobertura de } \\
\text { tallo } \mathrm{cm}^{2}\end{array}$ \\
\hline Mezquite & 82,76 & 99,86 & 99,07 \\
Palo fierro & 56,00 & 99,60 & 97,74 \\
Palo verde azul & 80,91 & 99,83 & 99,05 \\
Palo verde chino & 81,57 & 99,84 & 99,00 \\
\hline
\end{tabular}

De acuerdo a los parámetros climatológicos (Tabla 3), que se consideraron de gran importancia para este estudio, se observa que en los años 2016 y 2017 se presentaron las temperaturas máximas en promedio anual más altas de 34,1 a 34,4 grados centígrados, mientras que las temperaturas mínimas en promedio más altas se registraron en 2014 y 2015. Por otra parte, se presentaron en promedio anual las precipitaciones más altas en los años 2015 y 2018, con 401,83 mm y 431,62 mm, y por último, el porcentaje en promedio anual de la humedad relativa (HR) se registró en los años en que se presentaron los valores más altos de precipitación pluvial (PP) (Figura 3).

Tabla 3.-Promedio anual de las Temperaturas, Precipitación y \% de Humedad Relativa.

\begin{tabular}{ccccc}
\hline Año & Temp. Max $\left({ }^{\circ} \mathrm{C}\right)$ & Temp. Min $\left({ }^{\circ} \mathrm{C}\right)$ & PP mm & $\%$ HR \\
\hline 2010 & 32,5 & 15,1 & 324,01 & 46,83 \\
2011 & 33,4 & 14,6 & 309,32 & 46,11 \\
2012 & 33,8 & 15,3 & 296,68 & 44,92 \\
2013 & 33,0 & 14,8 & 317,45 & 46,92 \\
2014 & 33,8 & 16,3 & 312,57 & 45,72 \\
2015 & 33,9 & 16,2 & 401,82 & 47,24 \\
2016 & 34,1 & 15,3 & 283,35 & 44,79 \\
2017 & 34,4 & 16,0 & 282,55 & 44,47 \\
2018 & 31,2 & 13,6 & 431,62 & 48,94 \\
\hline
\end{tabular}

Temp. Max=Temperatura máxima, Temp. Min=Temperatura mínima, ${ }^{\circ} \mathrm{C}=$ Grados Centígrados, PP mm=Precipitación pluvial en mm y $\% \mathrm{HR}=$ Porciento de Humedad Relativa.

Cabe mencionar que las condiciones climatológicas presentes durante los últimos 8 años fueron óptimas para el desarrollo y establecimiento de las especies en estudio, al asegurar su perpetuidad, como en el caso del mezquite y ambos palos verdes, los cuales mostraron floración al $5^{\circ}$ año después de su trasplante y a su vez produjeron semillas, dando la 
posibilidad del reclutamiento de nuevas especies en el área de estudio. Los resultados obtenidos garantizan que a mediano plazo estas áreas perturbadas pueden volver a regenerarse con el uso de plantas nativas.

\section{Conclusiones y recomendaciones}

El tratamiento pregerminativo que se utilizó para cada una de las especies en este estudio presentó muy buenos resultados, lo cual es ventajoso. Además, el costo de dichos tratamientos es muy bajo, con lo cual es más económica la germinación.
El uso de plantas con altura superior a los $40 \mathrm{~cm}$ y con edad de cuatro meses presentó un efecto muy importante mostrando un porcentaje de adaptación a su nuevo hábitat.

Con respecto a las variables de altura, cobertura de copa y tallo, todas las especies obtuvieron muy buenos resultados después de los 8 años del trasplante, lo que permite esperar que, al llegar la primavera y las lluvias en verano, las plantas tendrán más fuerza y su desarrollo será mejor, elevando su índice de crecimiento.

\section{Literatura citada}

Baur. M.E.

2004. Pollution, Encyclopedia of Physical Science and Technology, 4rd Edition, Elsevier, Environmental Science Chapter. Environmental, University of California. Los Angeles, CA, US. 174 p.

Bos, G., Mastoris, J.

2011. Guidelines on Quarry Rehabilitation. World business counsil for sustentable development. Cement Sustainability Initiative (CSI). Switzerland. 29p.

Carrillo Flores, R.; Gómez Lorence, F.; Arreola Ávila, J. G. 2007. Efecto de poda sobre potencial productivo de mezquitales nativos en la comarca lagunera, México. Unidad Regional Universitaria de Zonas Áridas. Bermejillo, Dgo., Revista Chapingo Serie Zonas Áridas, 6: 47-54 p.

García M., G. E.; Jiménez, P. J.; Aguirre C. O. A.; González,

R. H.; Carrillo, P. A.; Espinosa, R. M.; García G., D. A.

2014. Biomasa de dos especies de matorral en tres densidades de plantación en Tamaulipas, México. Revista Latinoamericana de Recursos Naturales, 10 (2): 52-59 p.

International Seed Testing Association.

1999. International Rules for Seed Testing. Seed Science and Technology. 27-Supplement. ISTA. Switzerland. 333 pp.

Mc Caughey-Espinoza. D. M.; Ayala-Astorga, G.I.; Velázquez-

Caudillo, J.; Anaya-Islas, J.; Canseco-Vilches, E.

2017. Creación de un jardín botánico y de árbol madre de arbustivas forrajeras nativas del estado de Sonora. IDESIA Chile, 35 (4): 35-45.

Meza, S. R.; Osuna, E. L.

2003. Estudio Dasométrico del Mezquite en la Zona de Las Pocitas, B. C. S. INIFAP, Campo Experimental Todos Santos. México. La Paz, BC, México. 52 p.

México,

2010. Norma Oficial MEXICANA NOM-059SEMARNAT-2010. Protección ambiental-especies nativas de México de flora y fauna silvestre. Categoría de riesgo y especificaciones para su inclusión, exclusión o cambio de lista de especies en riesgo. Diario Oficial de la federación. 63 p.
Quiñoónez González, A.; González Ontivero, V.; Chávez Pérez, J.R.; Vargas Martínez, A.; Barrientos Díaz, F.

2013. Evaluación de inoculantes promotores de crecimiento en la producción de plántulas de mezquite (Prosopis laevigata (Humb. y Bonpl. Ex Willd) M. C. Johnston) en Durango. Revista Mexicana Ciencias Forestales, 4 (20): 13.

Ríos, S. J., Rivera G. M., Valenzuela, N. L., Trucios, C. R.; Rosales, S. R.

2011. Diagnosis of mesquite reforestation and methods for increasing survival in Durango, Mexico. Revista Chapingo serie Zonas áridas, 11 (2): 53-67.

Rueda-Puente, E.O.; Duarte-Medin, M.; Del Toro-Sánchez, C.; Murillo-Amado, B.; Ortega-Gracia J.; Rangel-Preciad, P.; Hernández-Montiel, L.G.; Borboa-Flores, J.; Wong-Corral, F.J. 2017 Efecto de la inoculación de Rhodococcus fascians y Azospirillum halopraeferens en la germinación de palo fierro (Olneya tesota A. Gray) en condiciones de invernadero. Tropical and Subtropical Agroecosystems, 20 (1): 11-18.

Sañudo-Torres, R.; Vázquez-Peñate, P.; Armenta-López, C.; Azpiroz Rivero, H.S.; Campos Beltrán, C.; Ibarra-Ceceña, M.G.; Félix- Herrán, J.A.

2009. Tratamientos pregerminativos en semillas de palo fierro (Olneya tesota A. Gray) y propagación en sustrato de composta de lirio acuático (Eichhornia crassipes). Ra Ximhai. Revista de Sociedad, Cultura y Desarrollo Sustentable, 5 (3): 1-6.

Sobrevilla Solís, J. A.; López Herrera, M.; López Escamilla A.; Romero Bautista, L.

2013. Evaluación de diferentes tratamientos pregerminativos y osmosis en la germinación de semillas Prosopis laevigata (Humb. y Bonpl. Ex Willd) M. C. Johnston. Estudios Científicos en el estado de Hidalgo y zonas aledañas, 12:82-95.90-91

Villarreal Garza, J. A.; Rocha Estrada, A.; Cárdenas Ávila, M.

L.; Moreno Limón, S.; Guzmán Álvarez, M. y Vargas López, V. 2013.Caracterización y germinación de semillas de mezquite y huizache en el noroeste de México. Revista internacional de botánica experimental OYTON, 82:169-174

Turner, R. M.; Bowers, J. E.; Burgess, T. L. 2005. Sonoran Desert plants: an ecological atlas. University of Arizona Press: Tucson, AZ, US. 501 p. 